\section{Journal of \\ Epilepsy and \\ Clinical \\ Neurophysiology}

J Epilepsy Clin Neurophysiol 2010;16(1):13-17

\title{
Mortalidade evitável: adequado manejo e assistência à saúde podem salvar vidas de pessoas com epilepsia?
}

\author{
Priscila Moraes de Paula Toledo Porto*, Paula Teixeira Fernandes*,**, \\ Carlos Alberto Mantovani Guerreiro*, Li Min Li*,**
}

Departamento de Neurologia - Faculdade de Ciências Médicas, UNICAMP

\begin{abstract}
RESUMO
Introdução: A mortalidade por epilepsia ainda é uma questão negligenciada e as mortes relacionadas com a epilepsia não são bem compreendidas. Recentemente, alguns estudos têm operacionalizado a metodologia de mortalidade evitável, descrevendo as causas de morte que não devem ocorrer na presença de boa qualidade de serviços e tratamento médico adequado. Esse debate sobre causas de morte evitáveis é amplo e parece promissor para o desenvolvimento do monitoramento e avaliação de desempenho da atenção à saúde de pessoas com epilepsia. Objetivo: explorar e selecionar os estudos que priorizaram a epilepsia como evento sentinela ou estudos de mortalidade evitável onde a seleção da epilepsia foi caracterizada dentro do grupo de causas evitáveis de morte. Metodologia: A revisão baseou-se numa pesquisa do banco de dados PubMed, usando os descritores, "mortality", "avoidable", "death" e "epilepsy", sem limites de tempo na data de publicação. Apenas artigos de particular relevância para a compreensão da morte evitável por epilepsia foram recuperados e revistos. Resultados: Encontramos uma variedade de estudos que incluíram a epilepsia dentro do grupo de causas de morte evitáveis e assim, pudemos discorrer a temática que pode nortear as ações e políticas públicas de saúde, facilitando a adoção de mecanismos de monitoramento do desempenho da atenção e da prevenção da saúde dos pacientes com epilepsia. Conclusões: Diante dos resultados encontrados, sugerimos como estratégias que poderiam ser consideradas pelos órgãos públicos de saúde, a implantação de comitês de mortalidade, que sejam de natureza interinstitucional, multiprofissional e confidencial para a prevenção da mortalidade relacionada à epilepsia. Com isso, medidas de intervenção podem ser realizadas para melhorar o atendimento das pessoas com epilepsia e, consequentemente, reduzir os óbitos relacionados à epilepsia.
\end{abstract}

Unitermos: Epilepsia, mortalidade, morte evitável, mortes reduzíveis.

\begin{abstract}
Avoidable death: Appropriate management and health care can save lives of people with epilepsy?

Introduction: The epilepsy avoidable death is a neglected issue. Deaths related to epilepsy are still not fully understood. Recently, some studies have showed the methodology of avoidable death, describing the causes of death should not occur in the presence of good quality services and appropriate medical treatment. The impact of studying the causes of avoidable death is relevant and promising for the monitoring and evaluation of performance of health care system for people with epilepsy. Purpose: To explore and select the studies emphasizing epilepsy as avoidable death or avoidable mortality studies where the selection of epilepsy was defined within the group of preventable causes of death. Methodology: The review was based on a search in the database PubMed, using as key words: "mortality", "avoidable", "death" and "epilepsy", without time limits on the date of publication. Results: We observed a variety of studies of epilepsy in the group of avoidable causes of death. So, we could discuss the topics that could guide the actions and public health policies. Conclusions: These results suggest strategies involving the establishment of committees of mortality - institutional, multidisciplinary and confidential - to prevent mortality related to epilepsy. So, the adequate intervention' strategies can be performed to improve the management of people with epilepsy and, consequently, to reduce the mortality on epilepsy.
\end{abstract}

Keywords: Epilepsy, mortality, avoidable death.

* Departamento de Neurologia - Faculdade de Ciências Médicas, UNICAMP.

** ASPE.

Received Nov. 23, 2009; accepted Dec. 18, 2009. 


\section{INTRODUÇÃO}

A epilepsia é a condição neurológica que afeta aproximadamente $1-2 \%$ da população mundial, sendo um dos problemas neurológicos mais frequentes (Sander, 2002). Existe consenso e evidência de que a epilepsia reduz a expectativa de vida, apesar de ter um bom prognóstico no que se refere ao controle de crises (Lathoo \& Sander, 2003). Uma meta-análise (Shackleton et al., 2002) mostrou que as taxas de mortalidade por epilepsia variam entre os estudos e demonstrou que a taxa de mortalidade padronizada (SMR) por epilepsia estava entre 1,3 e 9,3 vezes maior que a observada na população em geral.

Historicamente, a mortalidade por epilepsia é uma questão negligenciada e as mortes relacionadas com a epilepsia não são bem compreendidas (Sander \& Bell, 2003). Recentemente, a epilepsia vem sendo considerada como uma das principais causas evitáveis de morte, sendo que as potenciais falhas referem-se ao atendimento $\mathrm{e}$ tratamento de pessoas com epilepsia de maneira inadequada (Hanna et al., 2002). A morte por epilepsia é considerada um sinal de alerta, um "evento sentinela" que não deveria ocorrer se o serviço de saúde funcionar adequadamente (APPG, 2007). Esse debate sobre causas de morte evitáveis é amplo e parece promissor para o desenvolvimento do monitoramento e avaliação de desempenho da atenção à saúde.

Este artigo tem o objetivo de explorar os estudos publicados sobre o tema da mortalidade por epilepsia, com ênfase na evitabilidade desses óbitos. Exploraremos conjuntamente os estudos, selecionando aqueles que priorizaram a epilepsia como evento sentinela ou estudos de mortalidade evitável onde a seleção da epilepsia foi caracterizada dentro do grupo de causas evitáveis de morte. A revisão baseou-se numa pesquisa do banco de dados PubMed, usando os descritores, "mortality", "avoidable", "death" e "epilepsy", sem limites de tempo na data de publicação. Apenas artigos de particular relevância para a compreensão da morte evitável por epilepsia, foram recuperados e revistos.

\section{DEFINIÇÕES E INDICADORES}

A questão da qualidade do manejo e assistência à saúde tem recentemente suscitado reflexões e pesquisas para avaliar os serviços prestados em termos de qualidade. Alguns estudos têm operacionalizado a metodologia de mortalidade evitável, por ser este um indicador tradicional de desempenho. Descreve também as causas de morte que não devem ocorrer na presença de boa qualidade de serviços e tratamento médico adequado e que podem ser influenciados, pelo menos em parte, pelas políticas públicas de saúde e pelo comportamento dos pacientes. Essa classificação de morte evitável, também conhecida como 'evento sentinela', foi primeiramente proposta por Rutstein e colaboradores (1976), definida como a causa de morte para as quais há evidências de que cuidados médicos preventivos podem postergar ou impedir o avanço da doença ou a recorrência dos eventos.

Tobias e Jackson (2001) dividiram o conceito de morte evitável em três categorias: 1) mortalidade evitável primária, na qual as condições evitáveis ocorrem através da mudança de comportamento individual ou intervenção ao nível de políticas públicas de saúde; 2) mortalidade evitável secundária, referente às condições evitáveis que respondem à detecção precoce e ao tratamento, geralmente relacionadas à atenção primária de saúde; 3) mortalidade evitável terciária, onde condições de letalidade podem ser significativamente reduzidas por tratamentos médicos ou cirúrgicos em geral.

\section{Epilepsia como evento sentinela e indicador de desempenho}

Para monitorar o efeito e a qualidade dos serviços de saúde, foi elaborada uma lista internacional de doenças consideradas evitáveis (Rutstein et al., 1976), ou seja, aquelas para as quais existem tecnologias capazes de impedir sua ocorrência. Desde então, a validade e a aplicabilidade do conceito de mortalidade evitável como uma medida de efeito da atenção à saúde foram demonstradas em vários estudos conduzidos em países desenvolvidos (Poikolainen \& Skola, 1995). Uma redução nos níveis da mortalidade evitável foi relacionada com a efetividade dos serviços de saúde em seis países europeus, entre 1950 e 1980 (Charlton, 1986), e no Quebec, entre 1982 e 1990 (Pampalon, 1993). Esses estudos apresentam diferenças nos padrões e tendências em razão da seleção das causas estudadas.

No atlas de mortalidade evitável publicado na Nova Zelândia (Tobias e Jackson, 2001) foram atribuídos pesos relativos para a prevenção primária, secundária e terciária e a epilepsia, apresentando respectivamente os seguintes pesos: 0,0;0,9 e 0,1, denotando assim a importância da assistência à saúde de pacientes com epilepsia, principalmente a nível secundário para reduzir as mortes.

Na Austrália, a epilepsia é considerada como uma das principais causas evitáveis de morte, com potenciais falhas no atendimento e tratamento, e está no ranking das cinco maiores causas evitáveis nas idades de 15-29 (Piers et al., 2001). Em decorrência da mortalidade por epilepsia incidirem em maior número em pessoas jovens, o controle desses óbitos é imperioso na luta pela redução das mortes precoces e da perda de vidas em fase economicamente ativa.

Um inquérito realizado pelo All-Party Parliamentary Group (APPG, 2007) apoiado pelo Joint Epilepsy Council Reino Unido e Irlanda intitulado 'Wasted Money Wasted 
Lives', concluiu que aproximadamente $60 \%$ das mortes de crianças com epilepsia e $39 \%$ das mortes entre os adultos são "provavelmente ou potencialmente evitáveis". O relatório ainda afirma que o estigma social, o sigilo e a ignorância médica generalizada constituem um círculo vicioso que leva a um serviço de má qualidade a nível primário ou secundário. O desperdício de dinheiro em serviços inadequados de saúde provoca mortes desnecessárias e danos à qualidade de vida das pessoas com epilepsia.

\section{Epilepsia como evento sentinela no Brasil}

No Brasil, a epilepsia foi recentemente incluída pelo governo como evento sentinela na lista de causas evitáveis, assim como o acidente vascular cerebral, câncer de mama, útero entre outros, compondo as doenças reduzíveis por diagnóstico e tratamento precoce, com taxas de mortalidade que variaram entre $0,5 \%$ e 3,2\% do total de óbitos evitáveis por diagnóstico e tratamento precoce (Volochko, 2000; Ortiz, 2001; Malta e Duarte, 2007; Abreu et al., 2007). Controversamente, nos últimos 50 anos, as taxas de mortalidade não revelam redução do risco de morte por epilepsia, mesmo a despeito dos avanços significativos no tratamento cirúrgico e farmacológico da epilepsia. Denotase que a evitabilidade dessas mortes parece estar relacionada basicamente ao acesso e à qualidade dos serviços, bem como à acurácia do diagnóstico, sendo importantes para a redução das mortes por epilepsia.

$\mathrm{Na}$ Tabela 1 selecionamos alguns estudos que priorizaram a epilepsia como evento sentinela ou estudos de mortalidade evitável onde a seleção da epilepsia como evento sentinela foi caracterizada.

Tabela 1. Epilepsia como evento sentinela nos estudos de mortalidade evitável

\begin{tabular}{lcc}
\hline \multicolumn{1}{c}{ Autor } & Ano da publicação & País \\
\hline Poikolainen \& Skola & 1986 & Finlândia \\
Volochko & 2000 & Brasil \\
Tobias \& Jackson & 2001 & Nova Zelândia \\
Ortiz (Fundação SEADE) & 2001 & Brasil \\
Hanna et al (National Sentinel & 2002 & Reino Unido \\
Clinical Audit of Epilepsy) & & \\
Lip \& Brodie & 2002 & Glasgow-UK \\
Harsgreaves & 2002 & Londres -UK \\
Nolte \& McKee & 2003 & Alemanha \\
Stirbu et al. & 2006 & Holanda \\
Grant et al. & 2006 & Escócia \\
Korda et al. & 2006 & Austrália \\
Abreu et al. & 2007 & Brasil \\
Malta \& Duarte & 2007 & Brasil \\
Piers et al. & 2007 & Austrália \\
APPG - All Party & 2007 & Inglaterra \\
Parliamentary Group & & \\
\hline
\end{tabular}

\section{Manejo adequado da epilepsia e redução da} mortalidade

O manejo inadequado dos pacientes com epilepsia pode contribuir para um número significativo de mortes evitáveis relacionadas à epilepsia a cada ano. Uma auditoria nacional da mortalidade por epilepsia no Reino Unido (Hanna et al., 2002) estabeleceu que a cada ano cerca de 1.000 pessoas morrem como resultado da epilepsia, sendo metade dessas mortes consideradas evitáveis. Este estudo envolveu a análise especializada de 2412 registros oficiais de pessoas que tiveram morte relacionada à epilepsia entre os anos de 1999 e 2000. O relatório destaca os pontos fracos na prestação de serviços e exige uma atenção renovada à prevenção, investigação e gestão das mortes relacionadas à epilepsia (Figura 1). O manejo e o cuidado das pessoas com epilepsia foram considerados inadequados em $77 \%$ dos casos de crianças e $54 \%$ dos casos de adultos. O número elevado de mortes potencialmente evitáveis identificados neste relatório sugerem a necessidade de melhorias consideráveis na gestão da epilepsia. Em relação ao manejo da epilepsia, denota-se que todos os países podem ser considerados "emergentes".

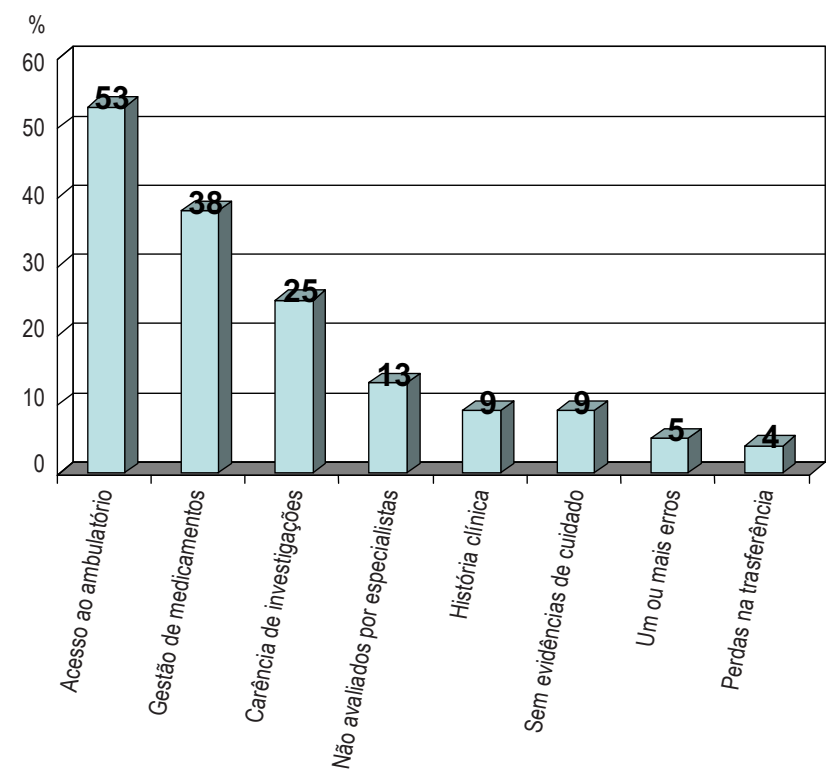

Figura 1. Fragilidades do sistema de atenção à saúde. Modificado de The National Sentinel Clinical Audit for Epilepsy Related Death"(Hanna et al., 2002).

Um estudo realizado no Brasil (Li et al., 2005), com o objetivo de fornecer uma visão global de como está a situação atual do manejo da epilepsia no Brasil, constatou que a porcentagem média de pessoas com epilepsia atendidas no último ano na rede básica de saúde está muito próxima a da prevalência estimada de epilepsia ativa no Brasil. Porém, alerta que para se estabelecer um efetivo programa de manejo adequado da epilepsia é necessária uma articulação 
da atenção básica da saúde com centros de referência secundários e terciários, uma distribuição contínua de drogas antiepilépticas e uma constante monitorização do manejo da epilepsia pelo governo brasileiro. Essas medidas podem contribuir na redução dessas mortes evitáveis.

Sander e Bell (2004) questionam sobre o que fazer para evitar essas mortes e quais melhorias na assistência e manejo podem ser implementadas. Também argumentam sobre a importância de realizar inquéritos de mortalidade relacionados com epilepsia nos países em que o cuidado é adequadamente percebido e onde o número de especialistas está de acordo com o tamanho da população, para determinar se a mortalidade é menor nesses contextos. Um número significativo de mortes entre pacientes com epilepsia poderia ter sido evitado se as normas recomendadas a nível nacional de cuidados fossem seguidas. Em seguida, conclamam: 'Chegou a hora primordial de reduzir a mortalidade através do adequado manejo da epilepsia' (Sander e Bell, 2004, p.350).

\section{Fatores determinantes de óbitos evitáveis na epilepsia}

Revisando estudos sobre eventos sentinela, é possível classificar cinco áreas prioritárias de investigação da morte, podendo aplicá-las inclusive às mortes por epilepsia (Tabela 2) para auxiliar na identificação e correção de falhas percebidas na cadeia de processos que deveriam prevenir danos a saúde da população.

Tabela 2. Determinantes de óbitos evitáveis na epilepsia

\begin{tabular}{ll}
\hline $\begin{array}{c}\text { Determinantes de } \\
\text { óbitos evitáveis }\end{array}$ & \multicolumn{1}{c}{ Fragilidade } \\
\hline $\begin{array}{l}\text { Comunidade e pessoa } \\
\text { com epilepsia }\end{array}$ & $\begin{array}{l}\text { Recusa em procurar assistência necessária } \\
\text { ou em seguir as orientações dos profissionais } \\
\text { de saúde, estigma associado ou por falta de } \\
\text { reconhecimento do problema } \\
\text { (Li et al., 2007). }\end{array}$ \\
& $\begin{array}{l}\text { Falta de capacitação ou capacitação } \\
\text { imprópria, negligência, imperícia, } \\
\text { imprudência ou falta de prioridade por parte } \\
\text { Profissionais }\end{array}$ \\
& pessoa com epilepsia (Sander \& Bell, 2004; \\
& Li et al., 2005). \\
& Problemas político-administrativos \\
contribuíram para o óbito, tais como & \\
carência de recursos ou medicamentos \\
e inexistência de sistema de referência \\
e contra-referência formalizado para o \\
tratamento clínico e cirúrgico da epilepsia, \\
entre outros \\
(Li et al., 2005). \\
Falta de equipamento social contribui para \\
o óbito. Por exemplo, disponibilidade \\
limitada de meios de acesso e transporte, \\
entre outros. \\
Casos em que as condições socioeconômicas \\
da pessoa com epilepsia contribuíram para \\
o óbito, tais como desemprego, baixa renda \\
familiar e baixa escolaridade da pessoa com \\
epilepsia, entre outros.
\end{tabular}

\section{Racismo institucional e mortalidade evitável}

"Saúde é direito de todos e dever do Estado", são os princípios da universalidade, equidade, integralidade e controle social da saúde, no entanto há indícios de desigualdades remanescentes na saúde. Teoricamente, com o manejo adequado, a morte por epilepsia é igualmente evitável para criança ou adulto, homem ou mulher; branco ou preto; economicamente desfavorável ou não. Porém, os serviços de saúde, intencionalmente ou não, contribuem para reproduzir a desigualdade racial na saúde, evidências mostram que ela não se associa à situação sócioeconômica do negro sugerindo a existência de racismo institucional, resultado do conjunto de políticas e normas de procedimentos e do comportamento dos membros das instituições (van Ryn e Fu, 2003).

Uma análise que aprofunda um projeto do governo brasileiro (Volochko, 2000), intitulado "Situação de saúde da população negra brasileira e recomendações para políticas, ações e programas", em parceira com a UNESCO, explorou a utilidade da mortalidade evitável como indicador capaz de desvelar as desigualdades na mortalidade por raça e gênero. $\mathrm{O}$ estudo revela que há indícios de que homens jovens negros com epilepsia são institucionalmente discriminados, tendo a epilepsia como a sétima causa de morte evitável com uma taxa 6,4 vezes maior que a de brancos, assim como a AIDS que também apresentou altas taxas de sobre mortalidade em relação aos brancos. Nas mulheres jovens negras com epilepsia a doença não aparece entre as principais causas evitáveis.

Corroborando com esses achados, uma revisão de literatura investigou as diferenças raciais e étnicas no tratamento da epilepsia e constatou uma subutilização dos serviços clínicos e tratamentos por essas minorias, que também demonstraram um conhecimento limitado sobre a epilepsia e seu tratamento, a falta de apoio social e a procura por terapias alternativas para a epilepsia (Szaflarski et al., 2006).

\section{CONCLUSÕES}

A epilepsia como evento sentinela ou morte evitável depende da disponibilidade dos recursos e da oportunidade de execução de medidas terapêuticas ou preventivas. Essa temática pode nortear as ações e políticas públicas de saúde, facilitando a adoção de mecanismos de monitoramento do desempenho da atenção e da prevenção da saúde dos pacientes com epilepsia.

Como estratégias que poderiam ser consideradas pelos órgãos públicos de saúde, sugerimos a implantação de órgãos que sejam de natureza interinstitucional, multiprofissional e confidencial para a prevenção da mortalidade relacionada à epilepsia, à semelhança de comitês de mortalidade, que 
teriam a função de determinar os óbitos relacionados à epilepsia, identificarem os fatores causais ou concorrentes e apontar medidas de intervenção para a redução dos óbitos relacionados à epilepsia na região de abrangência. Essas informações seriam então encaminhadas aos órgãos responsáveis pela saúde e educação e também aos profissionais diretamente ligados ao atendimento e assistência à saúde dos pacientes com epilepsia para melhorar o atendimento das pessoas com epilepsia e a consequente redução da mortalidade.

\section{REFERÊNCIAS}

1. Sander JWAS. Global Campaign Against Epilepsy. Overview of the demonstration projects. Epilepsia 2002;43(Suppl 6):34-6.

2. Lhatoo S, Sander JW. The prognosis of epilepsy in the National General Practice Study. In: Jallon P (Ed). Prognosis of Epilepsies. John Libbey; 2003. p. 64-72.

3. Shackleton DP, Westendorp RGJ, Kasteleijn-Nolst Trenite DGA, et al. Survival of patients with epilepsy: an estimate of the mortality risk. Epilepsia 2002;43:445-450.

4. Sander JW, Bell GS. Reducing mortality: an important aim of epilepsy management. J Neurol Neurosurg Psychiatry 2004;75:349-51.

5. Hanna NJ, Black M, Sander JWS, et al. National Sentinel Clinical Audit of Epilepsy-Related Death: Epilepsy - death in the shadows. London: The Stationery Office; 2002.

6. APPG - All Party Parliamentary Group on Epilepsy. The Human and Economic Cost of Epilepsy in England: Wasted Money, Wasted Lives. London: APPG on Epilepsy; 2007.

7. Rutstein DD, Berenberg W, Chalmers TC, Child CG, Fishman AP, Perrin EB. Measuring the quality of medical care: a clinical method. N Engl J Med 1976;294(11):582-8.

8. Tobias M, Jackson G. Avoidable mortality in New Zealand, 1981-97. Aust N J Z Public Health 2001;25(1):12-20.

9. Poikolainen K, Eskola J. The effect of health services on mortality: decline in death rates from amenable and non-amenable causes in Finland, 1969-81. Lancet 1986;1(8474):199-202.

10. Charlton J, Velez R. Some international comparisons of mortality amenable to medical intervention. British Medical Journal 1986;292:295-301.

11. Pampalon R. Avoidable mortality in Québec and its Regions. Social Science and Medicine 1993;37(6):823-831.

12. Piers LS, Carson NJ, Brown K, Ansari Z. Avoidable mortality in Victoria between 1979 and 2001. Australian and New Zealand journal of Public Health. Aust NZ Public Health 2007;31(1):5-12.
13. Volochko A. Mortalidade evitável na região Sul do Brasil, 2000: Desigualdades raciais e sexuais. Tese (Doutorado). Coordenadoria de Controle de Doenças, Secretaria de Estado da Saúde de São Paulo, 2005.

14. Ortiz LP. A mortalidade por causas evitáveis no estado de São Paulo - 1975/1976. Informe Demográfico (São Paulo: Fundação SEADE) 1980;4:49-109.

15. Malta DC, Duarte EC. Causas de mortes evitáveis por ações efetivas dos serviços de saúde: uma revisão da literatura. Ciência e Saúde Coletiva 2007;12(3):765-76.

16. Abreu DMX, César CC, França EB. Relação entre as causas de morte evitáveis por atenção à saúde e a implementação do Sistema Único de Saúde no Brasil. Rev Panam Salud Publica 2007;21(5):282-91.

17. Lip GY, Brodie MJ. Sudden Death in Epilepsy: An Avoidable Outcome? J R Soc Med 1992;85(10):609-11.

18. Hargreaves S. "Shortcomings in care" to blame for avoidable epilepsy deaths. BMJ 2002;324:1237.

19. Nolte E, McKee M. Does health care save lives? Avoidable mortality revisited. London: The Nuffiled Trust; 2004.

20. Stirbu I, Kunst AE, Bos V, Mackenbach JP. Differences in avoidable mortality between migrants and the native Dutch in the Netherlands. BMC Public Health 2006;6:78.

21. Grant I, Munoz-Arroyo R, Oduro S, Whyte B, Fischbacher C. Mortality amenable to health care in Scotland 1981-2004. Scottish Public Health Observatory Programme, Information Services Division. 2006.

22. Korda RJ, Butler JRG, Clements MS, Kunitz S. Differential impacts of health care in Australia: trend analysis of socioeconomic inequalities in avoidable mortality. Int J Epidemiol 2007;36:157-65.

23. Li LM, Fernandes PT, Noronha ALA et al. Demonstration Project on Epilepsy in Brazil: outcome assessment. Arq Neuropsiquiatr 2007;65(Supl 1):58-62.

24. Li LM, Fernandes PT, Mory S, Noronha ALA, Boer HMd, Espíndola J, Miranda C, Sander JW, Prilipko L. Manejo da epilepsia na rede básica de saúde no Brasil: os profissionais estão preparados? Rev Panam Salud Publica 2005;18(4-5):296-302.

25. Van Ryn M. Fu SS. Paved with good intentions: Do Public Health and Human Service Providers Contribute to Racial/Ethnic Disparities in Health? Am J Public health 2003;93(2):248-55.

26. Szaflarski M, Szaflarski JP, Privitera MD, Ficker DM, Horner RD. Racial/ ethnic disparities in the treatment of epilepsy: What do we know? What do we need to know? Epilepsy \& Behavior 2006 Sept;9(2):243-64.

Endereço para correspondência:

Li Li Min

Departamento de Neurologia - FCM - UNICAMP

Cx. Postal 6111

CEP 13083-970, Campinas, SP, Brasil

Tel.: (19) 3521-7292

E-mail:limin@fcm.unicamp.br 